\title{
Der Einfluß von Serumeiweißfraktionen auf die Fettsäuresynthese
}

\author{
In vitro-Untersuchungen mit dem löslichen Enzymsystem der Taubenleber ${ }^{1}$ )
}

Von W. SeItz und D. Eberhagen

Aus der Medizinischen Poliklinik der Universität München (Direktor: Prof. Dr. W. Seitz)

(Eingegangen am 7. Mai 1965)

\begin{abstract}
Drei Mischseren von Normalpersonen und zwei Diabetikerseren wurden mit Hilfe dẹ präparativen trägerfreien Elektrophorese aufgetrennt und die Einzelfraktionen entsprechend $4 \mathrm{mg}$ Eiweiß in vitro auf ihre Wirkung auf das nichtpartikelgebundene fettsäuresynthetisierende Enzymsystem der Taubenleber hin untersucht. Im Normalserum ließen sich dabei eine elektrophoretisch sehr rasch wandernde Fraktion mit ausgeprägter Förderung und zwei mit einer Hemmung des ${ }^{14} \mathrm{C}$-Einbaus aus Acetat in die langkettigen Fettsäuren nachweisen. In den Diabetikerseren fand sich außerdem eine stark hemmende Fraktion mit ebenfalls hoher Wanderungsgeschwindigkeit im elektrischen Feld, die als Ursache für die Repression der Fettsäuresynthese in der Diabetesleber diskutiert wird.

Um die verschiedenen Inkubationsansätze direkt miteinander vergleichen zu können, wurde bei den Untersuchungen ein Enzymtrockenpulver verwendet, das sich in großen Mengen gewinnen läßt, über längere Zeiträume lagerfähig ist und eine sehr einfache Durchführung der Syntheseverșuche gestattet.

Three mixed sera from normal persons and two diabetic sera were resolved by preparative, carrier-free electrophoresis. An amount of each fraction, corresponding to $4 \mathrm{mg}$. of protein, was tested for its action on the nonparticle-bound fatty acid synthesizing enzyme system of pigeon liver. Normal serum contains a fraction which migrates very rapidly in electrophoresis and shows a marked stimulation of the incorporation of ${ }^{14} \mathrm{C}$-acetate into long chain fatty acids. Two further fractions inhibited this incorporation. Diabetic sera contain, in addition, a strongly inhibitory fraction which migrates rapidly in electrophoresis and is thought to be responsible for the repression of fatty acid synthesis in diabetic liver.

In order to be able to directly compare the different incubation mixtures with each other, dry enzyme powders were used. These can be prepared in large quantities and stored for long periods of time; they greatly simplify the procedure for synthesis experiments.
\end{abstract}

Die letzten Jahre haben neue Erkenntnisse über die grundsätzlichen Mechanismen der Synthese langkettiger Fettsäuren in der Leber gebracht (1). Danach gibt es zwei verschiedene Enzymsysteme, von denen das eine in den Mitochondrien und Mikrosomen lokalisiert und nur zur Verlängerung bereits vorgegebener Fettsäuren um jeweils zwei C-Einheiten in der Lage ist (2). Das andere findet sich sowohl in den subzellulären Partikeln als auch ohne Bindung an Zellstrukturen im freien Zytoplasma (2-5). Es synthetisiert die Fettsäuren von Grund auf in zwei komplexen Schritten aus Acetyl-CoA. In der Acetylcarboxylase-Reaktion wird aus Acetyl-CoA durch Fixation von Bicarbonat-Ionen Malonyl-CoA gebildet; die anschließende Synthetase-Reaktion setzt $n$ Mol des entstandenen Malonyl-CoA unter Decarboxylierung mit einem Mol Acetyl-CoA um. Das Hauptprodukt der Gesamtreaktion ist Palmitinsäure. Man hat es hier also im Gegensatz zum partikelgebundenen Enzymsystem mit einer de novo-Synthese der Fettsäuren aus kleinsten, im Stoffwechsel aus anderen Quellen gelieferten Einheiten zu tun. $\mathrm{Da}$ die Syntheserate der langkettigen Fettsäuren - ausgedrückt als ${ }^{14} \mathrm{C}$-Einbau aus markiertem Acetat - im löslichen System etwa das Zehnfache der des mitochondralen und mikrosomalen Systems beträgt (1), ist es von ausschlaggebender Bedeutung für die Lipogenese und damit für die Energiespeicherung. Die Frage nach der Regulation seiner Aktivität hat dementsprechend vielseitiges Interesse gefunden. $\mathrm{MA}_{\mathrm{A}}$ sORo (6) hat eine Zusammenstellung der wichtigsten

1) Die Untersuchungen wurden durch Mittel der Deutschen Forschungsgemeinschaft unterstützt. bis 1961 bekannten Fakten gegeben. Er kommt zu dem Schluß, daß die Acetylcarboxylase-Reaktion der geschwindigkeitsbeschränkende Schritt der Gesamtreaktion ist, wie es sich z. B. aus den Untersuchungen von LYNEN und Mitarbeitern (7) ergab, und daß Hemm- und Förderstoffe eine wichtige Rolle bei der Regulation der Fettsäuresynthese spielen mögen.

Unser Arbeitskreis hat sich bereits in früheren Untersuchungen mit der Beeinflussung der zytoplasmatischen Fețăäuresynthese in der Leber beschäftigt (8-11), und es konnte aus der Taubenlebe $i$ ein aktivierender, hitzestabiler Faktor dargestellt werden (12). In einer vorausgehenden Arbeit (13) haben wir Ergebnisse mitgeteilt, die bei der Inkubation des Enzymsystems mit normalen und vor allem verschiedenen pathologischen Seren erhalten wurden. Es kam dabei in Abhängigkeit von der zugesetzten Serummenge zu einer Hemmung des ${ }^{14} \mathrm{C}$ Einbaus in die langkettigen Fettsäuren, deren Ausmaß in Beziehung zur jeweiligen, papierelektrophoretisch ermittelten $\alpha_{2}$-Globulinkonzentration stand. Kontrollinkubationen mit einem reinen $\alpha_{2}$-Makroglobulinpräparat ergaben ebenfalls eine drastische Hemmung der Fettsäuresynthese - im Gegensatz zu $\alpha_{1}$-Glukoproteid, das keinen Effekt zeigte. Uber den Angriffspunkt der Hemmung konnten keine Angaben gemacht werden. Diese Resultate bewogen uns, der Frage im einzelnen nachzugehen, welchen Effekt die verschiedenen Eiweißfraktionen des Serums auf die zytoplasmatische Fettsäuresynthese in vitro besitzen. Im Hintergrund steht die damit verknüpfte zweite Frage, ob es im Serum Faktoren gibt, die - evtl. aus der Leber selbst stammend - regulierend auf die Lipogenese wirken. 


\section{Versuche}

Die Herstellung des Enz'msystems erfolgte in Anlchnung an BradY und GURIN (3). Lebern 6-8 Monate alter, gut gefütterter Tauben wurden im Kaliumphosphat-bicarbonatpuffer ( $\mathrm{pH} 7,4)$ homogenisiert und der Gewebebrei erst 30 Minuten bei etwa $25000 \mathrm{~g}$, der Uberstand dann 75 Minuten bei $95000 \mathrm{~g}$ (Ultrazentrifuge Modell L der Fa. Beckman Instruments, München) zentrifugiert (Einzelheiten s. (10)). Die auf der Flüssigkeitsoberfiäche abgesetzte Fettschicht wurde sorgfältig entfernt und der Uberstand gefricrgetrocknct.

Der Inkubationsansatz enthielt in einem Endvolumen von $3,0 \mathrm{~m} /$ die jeweils $2,0 \mathrm{~m} /$ des Uberstandes entsprechende Menge Trockenpulver und $2 \mathrm{mg} 1{ }^{1.1} \mathrm{C}$-Acetat als $\mathrm{Na}-\mathrm{Salz}$ mit etwa $1,25 \times 10^{\mathrm{B}}$ Imp/Min. in der von uns verwendeten Mcßanordnung, sowic $4 \mu \mathrm{Mol} \mathrm{ATP}, 0,1 \mu \mathrm{Mol}$ NADPH, $0,04 \mu \mathrm{Mol} \mathrm{CoA}, 18 \mu \mathrm{Mol} \mathrm{Na}-$ Citrat und $18 \mu \mathrm{Mol} \mathrm{MgCl}_{2}$. Von den Serumfraktionen wurden dic $4 \mathrm{mg}$ Eiwciß entsprechenden Mengen eingesetzt. Es wurde 3 Stunden lang acrob in der Warburg-Apparatur inkubicrt. Dic Reaktion wurde dann durch Zugabe von $\mathrm{KOH}$ gestoppt und die Lipoide nach Zugabe von Trägerpalmitinsäure in alkoholischer Lösung verseift (14). Dic Fettsäuren wurden anschließend aus der angesäuerten Lösung mit $3 \times 20 \mathrm{~m} /$ Petroläther extrahicrt und die petrolätherischen Phasen durch Eindampfen und Wiederaufnehmen in Petroläther auf cin Volumen von $25 \mathrm{~m} /$ gebracht. Davon wurden $0,5 \mathrm{~m} /$ auf Kupferplättchen aufgetragen und nach Entfernen des Lösungsmittels dic Aktivität mit dem Methandurchflußzählrohr FH 407 der Fa. Frieseke \& Hocpfner, Erlangen-Bruck, bestimmt.

Die Anftrennung der Seren mit Hilfe der trägcrfreien Elektrophoresc geschah mit der von HanNig beschriebenen $\Lambda$ pparatur (15) (Fa. Bender \& Hobcin, München) in einem 0,02 $m$ Tris-Puffer pH 8,6 bei $+4^{\circ}$. Dic an dic Trennkammer angelegte Spannung betrug $2-2,2 \mathrm{kV}$ bei einem Strom von 150-180 mA. Die Durchflußgeschwindigkeit des Pufferstroms lag bei 0,8-0,9 cm/Min., dic Durchsatzleistung für das Scrum bei $1 \mathrm{~m} / / \mathrm{Std}$. Insgesamt wurden jeweils $4 \mathrm{~m} /$ Serum aufgetrennt. Der Eiweißgehalt in den 48 Auffanggläsern wurde durch Bestimmung der Extinktion bei $280 \mathrm{~m} \mu$ im Spektralphotometer DB der Fa. Beckman Instruments crmittelt.

Andere Methoden: Der genauc Eiwcißgehalt in den elektrophoretisch getrennten Serumfraktionen wurde mit der Biuretreaktion nach Werchselbaum (16), der Phosphorgehalt durch Veraschen von $0,2 \mathrm{~m} /$ Scrumfraktion nach BARTLETT (17) und der Kohlchydratgehalt mit der $\alpha$-Naphtholrcaktion nach Discire (18) bestimmt.
Zur weitcren Charakterisierung der Scrumeiwcißfraktionen wurde die Papicrelcktrophorese mit der Anordnung nach GrassmanN und HANNIG (19) und dic Immunpraczipitation nach OucirtekLONY (20) herangezogen.

Reagentien: Na-1-14C-Acetat wurde von der New England Nuclcar Corp., Boston USA gelicfert. CoA, NADPH und $\Lambda$ TP wurden von der Fa. Bochringer, Mannheim, Antiseren für dic Immunpraczipitation von der Fa. Bchringwerkc AG, Marburg-Lahn bezogen, die anderen Chemikalien von der Fa. E. Mcrck, Darmstadt.

\section{Ergebnisse}

Die Gewinnung des fettsäuresynthetisicrenden Enzymsystems

Bei der Bearbeitung der Fragestellung ergaben sich drei Forderungen an das zu verwendende Tcstsystem: 1 . Es mußtc jeweils eine relativ große Zahl von Proben untersucht werden. Der Arbeitsaufwand zur Bestimmung der ${ }^{14} \mathrm{C}$-Einbaurate sollte deshalb möglichst gering scin. 2. Dic verschicdenen Inkubationsansätze des gleichen Serums sollten dirckt miteinander vergleichbar sein. Dic Enzympräparation für eine derartigc Testreihe mußte also eine reproduzierbare Aktivität besitzen. 3. Das Enzymsystem sollte sich nach Möglichkcit ohne großen Geräte- und Arbcitsaufwand in genügender Reinheit und in ausreichenden Mengen darstellen lassen. Diese Forderungen waren am einfachsten zu erfüllen, wenn es gelang, die durch Ultrazentrifugation gewon-

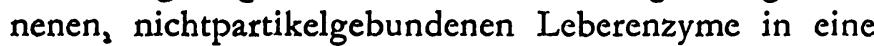
lagerfähige Form zu überführen. Hierzu kam in erster Linie die Herstellung eines Trockenpulvers durch Gefriertrocknung in Frage, denn das Aufbewahren großer Mengen von eingefrorenem Überstand über längere Zeiträume macht ohne besondere Hilfsmittcl Schwierigkeiten. Außerdem erschien die Erhaltung einer gleichbleibenden Enzymaktivität in gefrorenem Zustand nicht wie beim Trockenpulver gewährleistet (21).

Tab. 1

Verhalten der Aktivität des fettsäurcsynthetisicrenden Taubenleberenzymsystems vor und nach Lyophilisierung sowic bei Zusatz von Cofaktoren. (Inkubationsbedingungen siche Text.)

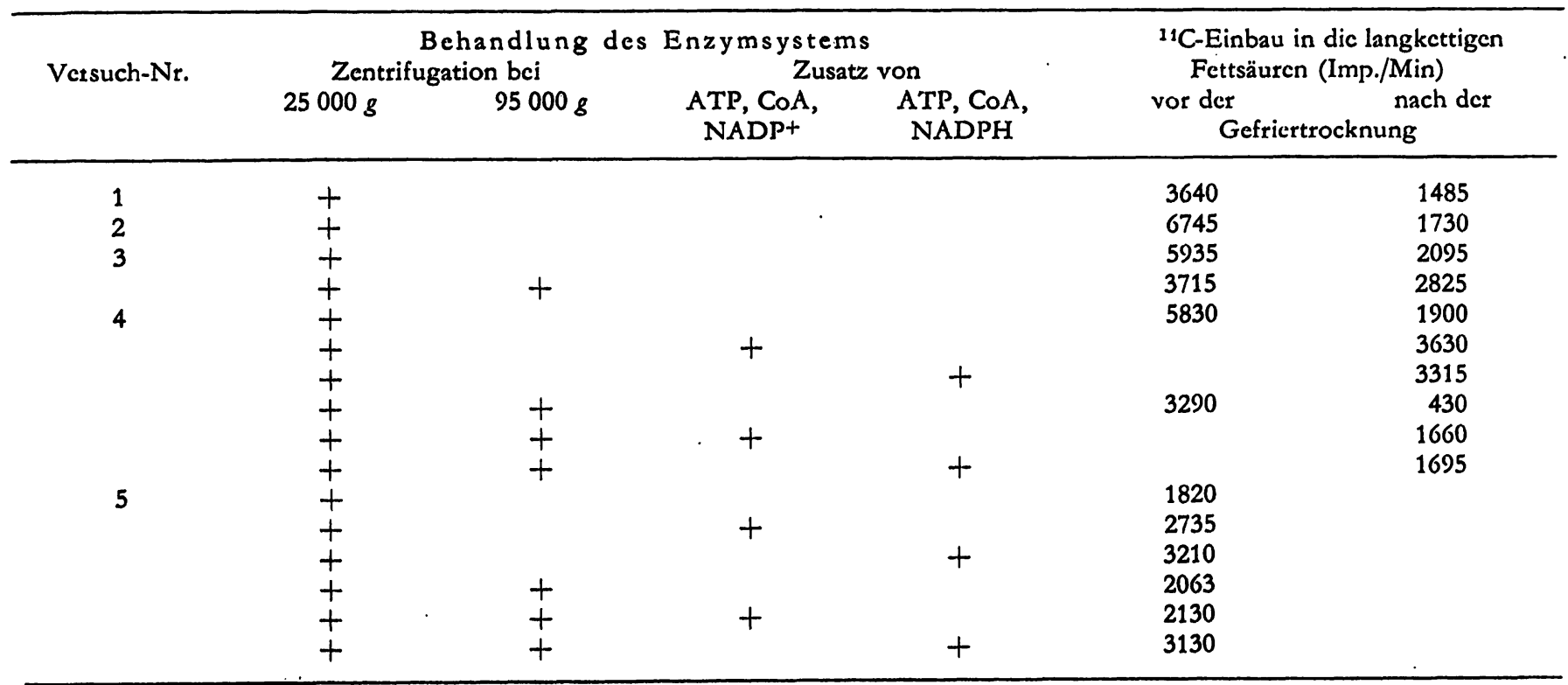


Eine wichtige Frage war es nun, ob das Enzymsystem eine solche Behandlung übersteht. Die Stabilität derartiger Fermentdarstellungen ist in der Literatur als sehr unterschiedlich angegeben (z. B. 21-23); trotzdem wurde eine Lyophilisierung des Materials versucht. Wie die Tabelle 1 an Hand einiger willkürlich ausgewählter Beispiele zeigt, trat durch diese Prozedur in jedem Fall ein erhellicher Aktivitätsverlust bis zu 50\% und mehr ein. Dabei schwankte das Ausmaß der Enzyminaktivierung außerordentlich, und Präparate mit primär höherem ${ }^{14} \mathrm{C}$-Umsatz schienen prozentual stärker denaturiert $\mathrm{zu}$ werden als solche mit niedrigem. Es wird von uns angenommen, daß diese Inkonstanz des Aktivitätsverlustes mit unserer improvisierten Gefriertrocknungseinrichtung zusammenhing.

Eine wesentliche Erhöhung der Syntheserate für die langkettigen Fettsäuren konnte durch Zusatz von ATP, CoA und NADP entsprechend dem von WAKIL (1) angegebenen Inkubationsansatz erreicht werden. Diese Erhöhung war, wie Versuche 4 und 5 der Tabelle 1 erkennen lassen, nicht durch eine Reaktivierung des denaturierten Anteils im Trockenpulver bedingt, sondern durch eine Stimulierung des Enzymsystems an sich. Die Zugabe dieser Cofaktoren zu den Ansätzen wurde im weiteren Verlauf der Untersuchungen außer wegen der Erhöhung des ${ }^{14} \mathrm{C}$-Einbaues schon deswegen beibehalten, um ähnliche Wirkungen durch die zu prüfenden Substanzen auszuschließen. Aus gleichem Grund wurde auch NADPH statt NADP + verwendet. Das als Trockenpulver hergestellte Präparat wurde in gleichem Ausmaß wie das Nativpräparat durch Avidinzusatz gehemmt; durch Biotin konnte diese Hemmung ebenfalls wieder aufgehoben werden.

Über die Lagerfähigkeit derartiger Trockenpulver unterrichtet die Tabelle 2. Die Präparate wurden im Vakuum über $\mathrm{CaCl}_{2}$ bei $+5^{\circ}$ aufbewahrt.

Tab. 2

Reproduzierbarkeit der Enzymaktivität eines Trockenpulvers Der zeitliche Abstand zwischen der exsten und der letzten Bestimmung betrug etwa 5 Wochen. Inkubationsbedingungen siehe Text.

${ }^{14} \mathrm{C}$-Einbau in die langkettigen Fettsäuren (Imp./Min.) vor der Gefriertrocknung

\begin{tabular}{rr}
\hline 1580 & 890 \\
& 1320 \\
& 1070 \\
& 920 \\
& 1100 \\
& 1210 \\
\hline
\end{tabular}

Es konnte keine Abnahme der Enzymaktivität während einer - bisher untersuchten - Dauer von maximal 5 Wochen beobachtet werden. Nach diesen Ergebnissen erschien die Darstellung des Fermentsystems als Trockenpulver im Rahmen unserer Untersuchungen als sehr geeignet; der Aktivitätsverlust während der Gefriertrocknung wurde bei den Vorteilen eines derartigen Präparates in Kauf genommen.
Die Inkubation mit Normalserum

Zur Auftrennung durch die trägerfreie Elektrophorese kamen bei der Untersuchung des Blutserums von Normalpersonen ausschließlich Mischseren zur Verwendung. In der Abbildung 1 sind die erzielten Trenneffekte dargestellt. Bei normaler Einstellung der Apparatur (Abb. $1 a$ und $b$ ) ließen sich auf Grund der Tyrosinextinktion fünf Fraktionen unterscheiden. Am weitesten zur Anode abgelenkt waren als anscheinend vollständig abgetrennte Fraktion die Präalbumine (Rö.-Nr. 7-9, Abb. 1a; Rö.-Nr. 4 und 5, Abb. 1b). Ihre Identität wurde durch Immunpräzipitation mit spezifischem Antiserum bestätigt. Albumine fanden sich in den Auffanggläsern 10-22 (Abb. 1 a) bzw. 7-19 (Abb. 1b). Dabei ließen sich sowohl papierelektrophoretisch als auch mit Hilfe der Immunpräzipitation in diesen Gläsern - wenn auch meistens nur in sehr getingen Mengen $-\alpha_{1}$-Globuline nachweisen. Große Anteile $\alpha_{2}$-Makro- . globulin waren in den Röhrchen 20-22 (Abb. 1a) bzw. 17-19 (Abb. 1b). Die Eiweißstoffe der Rö.-Nr. 25-29 (Abb. 1a) bzw. 21-24 (Abb. 1b) waren reine $\beta$-Globuline, die der Rö.-Nr. 32-40 (Abb. 1a) bzw. 27-34 (Abb. $1 \mathrm{~b}$ ) reine $\gamma$-Globuline.

Zur Inkubation mit dem fettsäuresynthetisierenden Enzymsystem wurde jeweils die $4 \mathrm{mg}$ Eiweiß enthaltende Lösungsmenge der Röhtchen eingesetzt. Als Bezugswert für die normale Höhe der Fettsäuresynthese liefen bei jedem Testansatz zwei Kontrollgefäße ohne einen derartigen Zusatz mit. Der in diesen Gefäßen festgestellte ${ }^{14} \mathrm{C}$-Einbau in die Fettsäuren wurde als $100 \%$-Wert angesehen und die anderen Impulszahlen darauf bezogen. Zuvor aber war sichergestellt worden, $\mathrm{da} \beta$ der bei der elektrophoretischen Auftrennung der Seren verwendete Tris-Puffer keinen Einfluß auf die Fettsäuresynthese besaß.

Überraschenderweise fand sich nun in den Auffanggläsern vor der Präalbuminfraktion ein Bereich mit einèr sehr stark ausgeprägten Förderung der Syntheserate. Die Biuretreaktion hatte hier hohe Eiweißwerte angezeigt, obwohl die Extinktion bei $280 \mathrm{~m} \mu$ nicht über dem Leerwert lag und auch papierelektrophoretisch selbst bei kurzer Elektrophoresedauer weder mit der Amidoschwarz- noch mit der Ninhydrinfärbung Komponenten nachweisbar waren. Parallel mit dem Ausmaß der Syntheseförderung ging, wie Abbildung $1 \mathrm{~b}$ zeigt, der Phosphorgehalt in den Röhrchen. Bei einer später durchgeführten Isolierung der in diesen Gläsern befindlichen Substanzen ergab sich ein weißes, sehr hygroskopisches Material, dessen weitere Charakterisierung noch aussteht. Die Präalbumine hatten keinen Einfluß auf die Fettsäuresynthese. In den Röhrchen unmittelbar vor den Albuminen waren Verbindungen mit synthesehemmenden Eigenschaften enthalten. İhre Wanderungsgeschwindigkeit im elektrischen Feld lag zwischen der der Albumine und der der Präalbumine. Bei einer Auftrennung mit einem gegenüber den Standardbedingungen beschleunigten Pufferstrom, bei welcher also die Einwirkung des elektrischen Feldes und damit die Ablenkung der Eiweißkörper quer zur Strömungsrichtung 


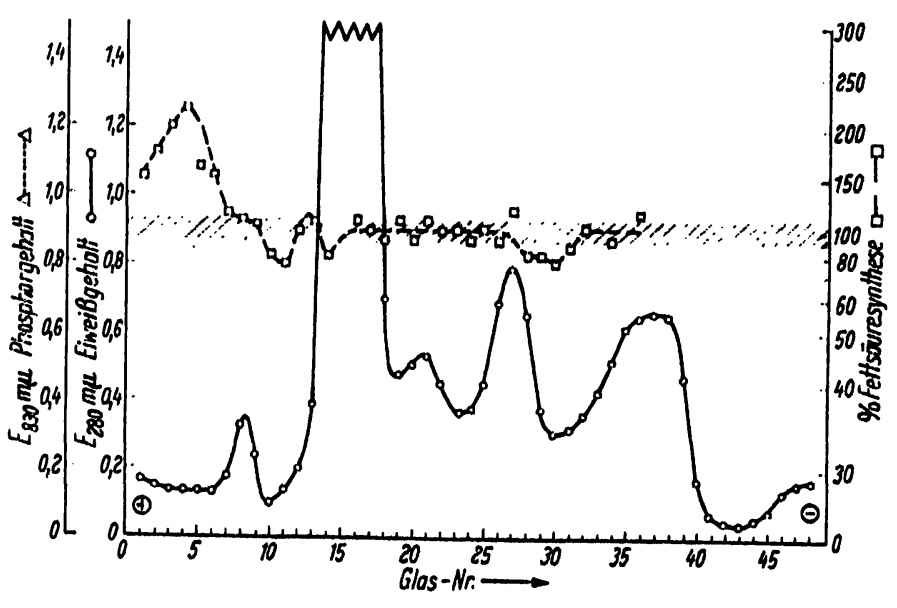

$\Lambda$ bb. 1 a

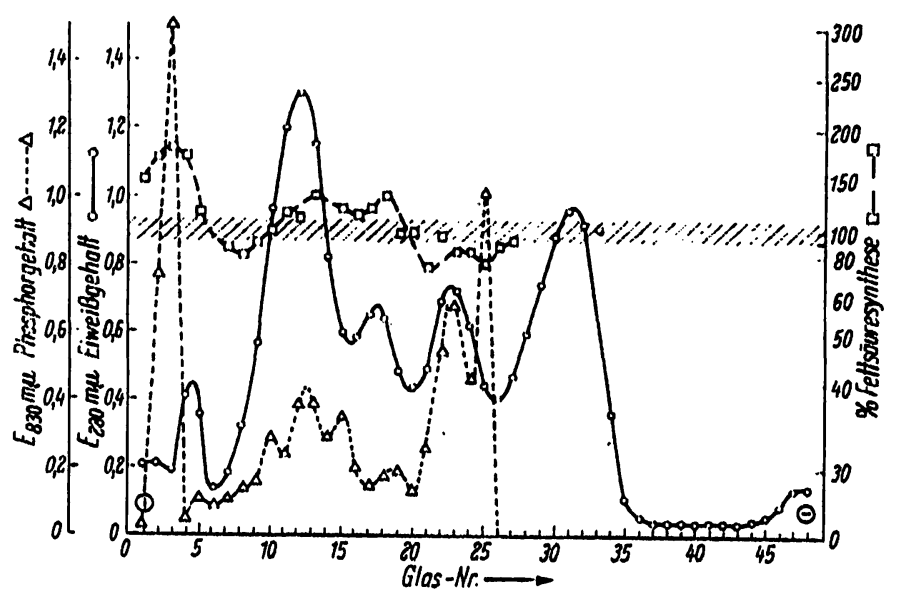

Abb. $1 \mathrm{~b}$

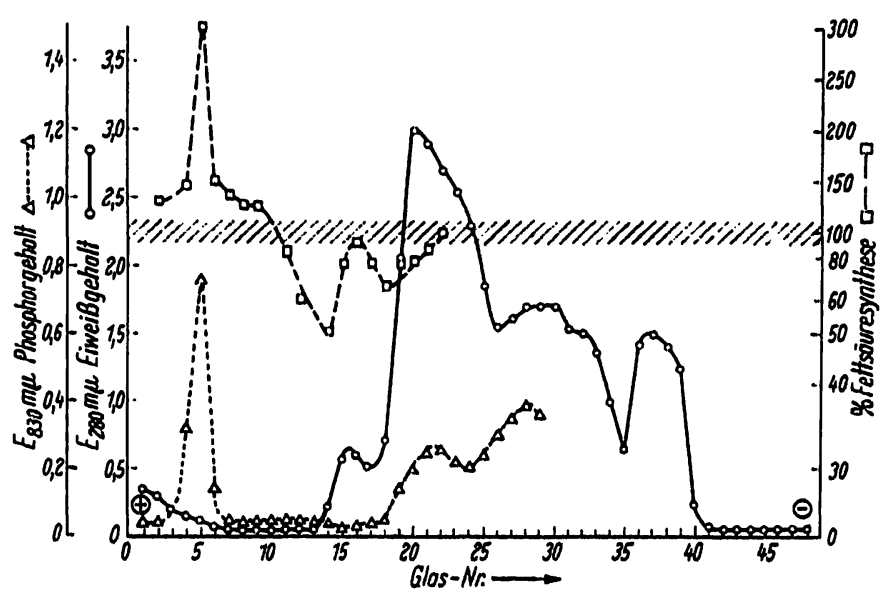

Abb. $1 \mathrm{c}$

$\Lambda$ bb. $1 \mathrm{a}-\mathrm{c}$

Becinflussung der zytoplasmatischen Fettsäuresynthese in der Taubenleber $(\square--\square)$-ausgedrückt als ${ }^{14} \mathrm{C}$-Einbau aus markicrtem Acetat in die langkettigen Fettsäuren - durch clektrophoretisch aufgetrennte EiwciBfraktionen von menschlichen Normalseren

Die Ordinate ist linear in Vielfachen der ungestörten Syntheserate aufgetragen, der 100\%-Bereich durch Schrägschrafficrung hervorgchoben. Der Eiweißgehalt der Fraktionen $(\mathrm{O}-\mathrm{O})$ ist als Extinktion bei $280 \mathrm{~m} \mu$ angegeben, der Phosphorgechalt $(\Delta \ldots \Delta)$ als Extinktion der nach Veraschung der Substanz gebildeten Phosphormolybdänsäure bei $830 \mathrm{~m} \mu$. Dic Scren sind in Höhe des Auffangglases Nr. 38 in dic Trennkammer cingeführt. verringert war, spaltete sich dicscr hemmende Bereich in zwei auf (Abb. 1c). Der fördernde Bereich mit den clektrophorctisch am schnellsten wandernden Substanzen überdecktc sich auch hicr wicder kongruent mit dem Bereich hohen Phosphorgehaltes. Besondere Aufmerksamkcit wurcle weitcrhin dem $\alpha_{2}$-Globulinbercich geschenkt, da - wic bereits cingangs crwähnt in früheren Untersuchungen mit cinem reinen $\alpha_{12}-$ Makroglobulinpräparat der Fa. Behring werke cin starker Hemmeffekt beobachtet worden war (13). Es konnte aber bei keinem der aufgetrennten Normalseren cine solche Wirkung auf das Enzy'msystem festgestellt werclen.

\section{Dic Inkubation mit Diabctikerserum}

In der Abbildung 2 ist dic clcktrophorctische Auftrennung zweier Diabctikerseren wiedergegeben. Bei der Abbildung 2 a handelt cs sich um das Scrum cincr 67jährigen Frau mit unbehandcltem Altersdiabetes (Blutzucker $170 \mathrm{mg} \%$, Harnzucker $6 \mathrm{~g} /$ Tag), bei der Abbildung $2 \mathrm{~b}$ um clas ciner 40 jährigen, bisher. unbehandelten Diabetikerin (Blutzucker $230 \mathrm{mg} \%$, I-Iarnzucker $3 \mathrm{~g} /$ Tag). Wieder fand sich im Bercich vor den Präalbuminen eine starke Förderung und in den Auf-
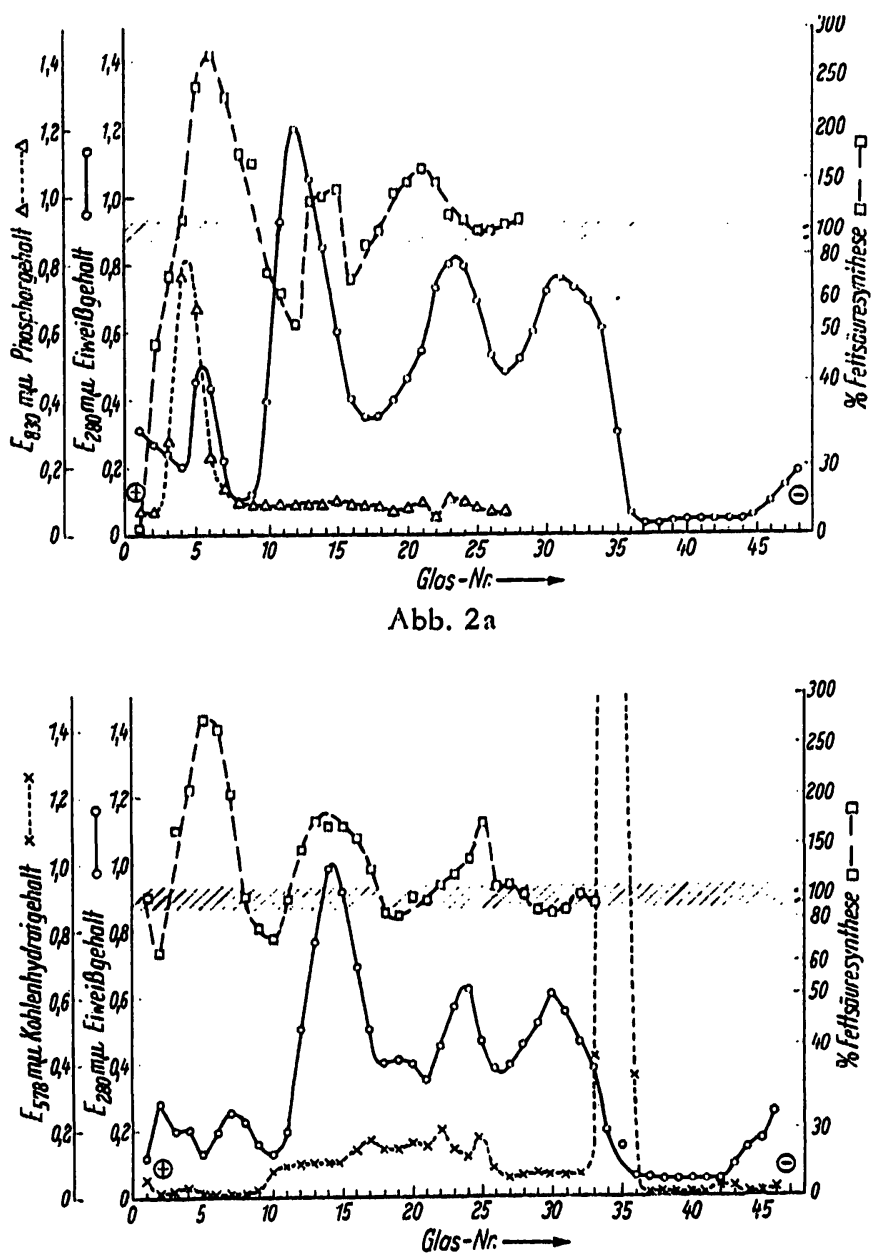

$\Lambda$ bb. $2 b$

Abb. $2 a$ und $b$

Becinflussung der zytoplasinatischen Fettsäurcsynthese in der Taubenleber $(\square--\square)$ clurch elektrophoretisch aufgetrennte Eiweißfraktionen von menschlichen Diabetikerseren. (Weitere Errklïrung siche Abbildung 1) 
fanggläsern $z$ wischen den Präalbuminen und den Albuminen eine Hemmung der Fettsäuresynthese. Bemerkenswerterweise kam es nun hier durch die im Bereich der $\alpha_{2}$-Globuline vorhandenen Eiweißstoffe zu einer Herabsetzung der Syntheserate, wie sie nach den erwähnten früheren Befunden auch ursprünglich erwartet wurde. Der auffälligste Unterschied zu den Normalseren aber war die Tatsache, daß in den Diabetikerseren noch ein weiterer Stoff mit starker Hemmwirkung enthalten war. Er fand sich jeweils in den ersten Röhrchen bei der von uns gewählten Anordnung, wurde also durch das elektrische Feld noch stärker als die fördernde Komponente abgelenkt. Wie die Abbildung 2a zeigt, verringerte sich dann mit zunehmendem Phosphorgehalt in den Röhrchen der hemmende Einfluß auf die Fettsäuresynthese, um dann in eine Förderung überzugehen. Das stützt unsere Vermutung, daß der Stoff mit Stimulatorwirkung einen hohen P-Gehalt besitzen muß. Die fördernde Wirkung der Albumin- und $\beta$-Globulinfraktionen wird als unspezifischer Effekt durch Bereitstellung von Akzeptoren für die neugebildeten Fettsäuren o. ä. angesehen. Der Kohlehydratgehalt - bestimmt mit der $\alpha$-Naphtholreaktion sowohl bei $546 \mathrm{~m} \mu$ (für Pentosen) als auch bei $578 \mathrm{~m} \mu$ (für Hexosen) - stand in keinem erkennbaren Zusammenhang mit der Wirkung der Fraktionen auf die Fettsäuresynthese (Abb. 2b).

Die Auffanggläser jeder Auftrennung wurden als Serie dreimal hintereinander auf ihre Beeinflussung der Fettsäuresynthese hin untersucht. Die Resultate entsprachen sich jeweils; das Ausmaß ihrer Wirkung verringerte sich leicht in Abhängigkeit zur Aufbewahrungsdauer der Elektrophorese-Fraktionen.

\section{Diskussion}

Die einzelnen Reaktionsschritte und Intermediärprodukte bei der Synthese der langkettigen Fettsäuren in der Leber und den anderen Organen scheinen heute in den wesentlichen Zügen bekannt $\mathrm{zu}$ sein. Die größte Bedeutung für die Neubildung von Fettsäuren hat nach allen zur Zeit verfügbaren Befunden das im Zytoplasma ohne Bindung an die subzellulären Strukturen lokalisierte Enzymsystem; jedoch ist nur wenig über physiologische Regulatoren seiner Aktivität und damit der Lipogenese bekannt. Sind derartige Substanzen mit fördernder oder hemmender Wirkung auf die Fettsäuresynthese in den Organen vorhanden, so dürften sie zumindest bei der Zellmauserung ins Blutplasma übertreten und dort nachweisbar werden. Diese Überlegung leitete uns bei unseren vorliegenden Untersuchungen, und in dieser Weise möchten wir unsere Ergebnisse interpretieren, obwohl sie - da wir die chemische Natur der einzelnen wirksamen Substanzen nicht kennen und ihre Wirkung auch noch nicht in vivo nachgeprüft haben - durchaus andere Deutungsmöglichkeiten zulassen. So ist beispielsweise von verschiedenen Untersuchergruppen $(24-27)$ über ähnliche Wirkungen von Sulfhydrylgruppen enthaltende oder bindende Substanzen berichtet worden.
Insgesamt konnten im Normalserum eine die Fettsäuresynthese in vitro fördernde und eine, evtl. sogar zwei hemmende Fraktionen festgestellt werden. Die fördernde Fraktion mit hohem Phosphorgehalt gab eine starke Biuretreaktion. Sie absorbierte nicht bei $280 \mathrm{~m} \mu$, wohl aber bei $230-240 \mathrm{~m} \mu$ und war nicht dialysierbar. Ihr Kohlehydratgehalt mußte sehr gering sein. Ihre Wanderungsgeschwindigkeit im elektrischen Feld war im Vergleich mit den Eiweißstoffen des Serums hoch. Diese Eigenschaften lassen am ehesten auf ein Polypeptid schließen; allerdings läßt sich die mangelnde Anfärbung mit Ninhydrin damit nicht vereinbaren. Die hemmende Fraktion, die elektrophoretisch unter den Standardbedingungen $z$ wischen den Präalbuminen und den Albuminen wanderte, scheint ebenfalls keine bisher bekannte oder in dieser Weise nachgewiesene Substanz zu sein. Die ursprüngliche Annahme - wie sie die Abb. $1 \mathrm{a}$ und $\mathrm{b}$ nahelegen - daß es sich hier um ein im elektrischen Feld schnell wanderndes Albumin handelt, mußte aufgegeben werden, als ein Serum unter anderen Bedingungen aufgetrennt wurde (Abb. 1c). Hierbei zeigte der hemmende Bereich eine weit stärkere $\mathrm{Ab}$ lenkung zur Anode hin als die Albumine; nur die zweite Hemmfraktion im ansteigenden Schenkel der Albumine könnte in dieser Weise zugeordnet werden.

Beide Fraktionen mit förderndem und hemmendem Einfluß auf die Fettsäuresynthese wurden auch in den Diabetikerseren gefunden. Außerdem ließ sich aber noch eine weitere mit der stärksten bisher beobachteten Hemmwirkung nachweisen, die keinen oder einen nur sehr niedrigen P-Gehalt, keine Absorption bei $280 \mathrm{~m} \mu$ und die größte elektrophoretische Beweglichkeit aller Substanzen besaß. Das Vorhandensein dieser Fraktion möchten wir mit der Beobachtung in Verbindung bringen, daß die zytoplesmatische Fettsäuresynthese in der Diabetesleber gegenüber der gesunden stark verringert ist (28-30). Vielleicht handelt es sich um den Faktòr, der verantwortlich für diese Repression ist. Im Gegensatz zu den Normalseren wurde hier durch die Eiweißstoffe des $\alpha_{2}$-Bereiches eine, wenn auch nicht sehr ausgeprägte, Hemmwirkung festgestellt. Diese Hemmwirkung hatten wir ursprünglich auch im Normalserum erwartet, da ein reines $\alpha_{2}$-Makroglobulinpräparat in früheren Untersuchungen (13) ebenfalls als Inhibitor in unserem Testsystem gewirkt hatte. Wir möchten daher für das Diabetikerserum das Vorhandensein eines $\alpha_{2}$-Globulins annehmen, das im normalen Blut nicht oder nur in wesentlich geringerer Konzentration enthalten ist.

Unsere oben mitgeteilten Ergebnisse lassen noch keine weitgehenden Deutungen zu. Erst wenn weitere Anhaltspunkte für die chemischen Strukturen, über die Art ihrer Wirkungsmechanismen und über ihre Wirksamkeit in vivo vorliegen, können Aussagen über ihre physiologische Bedeutung gemacht werden.

Frl. C. STIEHLER sind wir für ihre sorgfältige und einsatzfreudige Mitarbeit bei der Durchführung der Untersuchungen zu großem Dank verpflichtet. Weiterhin danken wir Frl. cand. med. A. Prowe und Herrn cand. med. S. HolzBerg für ihre Mitarbeit. 


\title{
Literatur
}

1. WAkIL, S. J., J. Lipid Res. 2, 1 (1961). -2. HARLAN jr., W.R. und S. J. Wakit, J. biol. Chemistry 238, 3216 (1963). - 3. Brady, R. O. und S. Gurin, J. biol. Chemistry 199, 421 (1952). - 4. Diruri, F. und S. Gurin, Arch. Biochem. Biophysics 43, 231 (1953). - 5. Van Banlen, J. und S. Gurin, J. biol. Chemistry 205, 303 (1953). - 6. MAsoro, E. J., J. Lpid Res. 3, 149 (1962). 7. Numa, S., M. Matsuriashi und F. Lynen, Biochem. Z. 334, 203 (1961). - 8. Seitz, W. und V. von Brand, Zschr. inn. Med., Leipzig 13, 764 (1958). - 9. Seitz, W. und V. von Brand, Klin. Wschr. 39, 891 (1961). - 10. von Brand, V., Biochem. Z. 331, 162 (1959). - 11. von Brand, V. und W. SeItz, Med. Klinik 55, 723 (1960). - 12. von Brand, V. und W. Sertz, Klin. Wschr. 36, 587 (1958). - 13. NAgengast-von Brand, V. und W. Seit', Klin. Wschr. 43, 402 (1965). - 14. von Brand, V. und E. Helmreici, Biochem. Z. 328, 146 (1956). - 15. Hannig, K. Z. analyt. Chem. 181, 244 (1961). - 16. Weichselbaum, T. E., Amer. J. Clin. Path. 10, 49 (1946). - 17. BArtlett, G. R., J. biol. Chemistry 234, 466 (1959). - 18. Discre, Z., in: D. Glick, Methods of Biochemical
Analysis. Bd. 2, S. 320. Interscience Publ., New York-London 1955. - 19. Grassmann, W. und K. Hannig, Klin. Wschr. 32, 838 (1954). - 20. Ouchterlony, O., Progr. Allergy 6, 30 (1962). - 21. Brady, R. O., R. M. Bradefy und E. G. Trams, J. biol. Chemistry 23.5, 3093 (1960). - 22. Tietz, A., Biochem. biophysica Acta (Amsterdam) 25, 303 (1957). - 23. Martin, D. B., M. G. Horning und P. R. Vagelos, J. biol. Chemistry 236, 663 (1961). - 24. Brady, R. O., A.-M. Mamoon und E. R. Stadtalan, J. biol. Chemistry 222, 795 (1956). - 25. BRADY, R. O., J. biol. Chemistry 235, 3099 (1960). - 26. Brady, R. O., E. G. Trams und R. M. Bradley, Biochem. biophysic Res. Commun. 2, 256 (1960). 27. Bressler, R. und S. J. Wakil, J. biol. Chemistry 237, 1441 (1962). - 28. Shaw, W. N., F. Dituri und S. Gurin, J. biol. Chemistry 226, 417 (1957). - 29. Auraham, S., K. J. Matthes und I. L: Charkoff, J. biol. Chemistry 235, 2251 (1960). - 30. Matthes, K. J., S. Abraham und I. L. Cilaikoff, J. biol. Chemistry $235,2560(1960)$.

\section{Semiquantitative, dünnschichtchromatographische Bestimmung der Gallensäuren im Serum, Duodenalsaft und Stuhl}

\author{
Von H.-Ch. Curtrus \\ Aus dem Mediqinisch-cbemischen Laboratorium der Universitäts-Kinderklinik Zïrich (Direktor: Prof. Dr. A. Prader) ${ }^{1}$ )
}

(Eingegangen am 4. Mai 1965)

\begin{abstract}
Es wird eine dünnschichtchromatographische Methode für die semiquantitative Bestimmung der Gallensäuren und deren Konjugate bei gesunden Erwachsenen beschrieben. Die Methode kann auch für die Bestimmung der Gallensäuren im Duodenalsaft und im Stuhl verwendet werden.

A thin layer chromatographic method is described for the semiquantitative determination of bile acids and their conjugates in healthy adults. The method can also be used for the determination of bile acids in duodenal juice and faeces.
\end{abstract}

Im menschlichen Serum sind bis jetzt Cholsäure, Desoxycholsäure und Chenodesoxycholsäure nachgewiesen und quantitativ bestimmt worden. Diese drei Gallensäuren kommen in freier wie auch in konjugierter Form vor. Bei den Konjugaten besteht eine Peptidbindung zwischen der Carboxylgruppe der Gallensäure einerseits und der Aminogruppe von Taurin bzw. Glycin andererseits.

Für die quantitative Bestimmung müssen die Gallensäuren aus dem Serum von den Eiweißen getrennt und isoliert werden. Die am häufigsten verwendete Methode besteht in der Fällung der Eiweiße mit äthanolischer Bariumhydroxyd-Lösung, die mit Bariumacetat gesättigt ist, nach JosephsoN (1). Aus dem Uberstand werden die Lipide mit Essigester extrahiert. Die Gallensäuren bleiben, weil sie als Bariumsalze vorliegen, in der wässerigen Phase zurück und können mit Furfurol/Schwefelsäure in einen Farbkomplex überführt und photometrisch bestimmt werden. - Bessere Resultate werden erzielt, wenn die Gallensäuren nach der Extraktion photometrisch im UV-Bereich (2) bei $350 \mathrm{~m} \mu$ oder fluori-

1) Der größte Teil der experimentellen Arbeiten wurde im Med.Chem. Zentrallaboratorium des Kantonsspitals Zürich (Leiter: P. D. Dr. H. RosenMund) ausgeführt. metrisch (3) bei $490 \mathrm{~m} \mu$ gemessen werden. Da sich die Absorptionsspektren von Desoxycholsäure und Chenodesoxycholsäure in Äthylacetat, Schwefelsäure, Essigsäureanhydrid und Salicylaldehyd-Schwefelsäure-Eisessig voneinander unterscheiden, können auch - mit gewissem Vorbehalt - Aussagen über die quantitative Verteilung der beiden Einzelkomponenten gemacht werden.

Die Extraktionsmethode nach Josephson weist verschiedene Nachteile auf. Die im Vergleich zu den Gallensäuren in viel größerer Menge vorhandenen Lipide, wie Cholesterin, Phosphatide und Monoglyceride werden nicht quantitativ entfernt und stören die nachfolgende Bestimmung. Dasselbe gilt für andere Extraktionsverfahren $(4,5)$. Außerdem entsteht gerade durch die Extraktion ein nicht zu vernachläßigender Verlust vor allem an freien Gallensäuren. Aus diesem Grunde ist es verständlich, daß die in der Literatur angegebenen Normalwerte für Gallensäuren im Serum zwischen 0 und $50 \mathrm{mg} \%$ schwanken.

In jüngster Zeit ist eine Arbeit von B. Frosch (6) erschienen, in der die konjugierten Gallensäuren nach Abtrennung der Eiweiße mit Äthanol und Extraktion 